\title{
Experimental Investigation of Polarization Effects in Semiconductor Optical Amplifiers and Implications for All-Optical Switching
}

\author{
Severine Philippe, A. L. Bradley, B. Kennedy, F. Surre, Member, IEEE, and Pascal Landais, Member, IEEE
}

\begin{abstract}
A free-space contra-propagation configuration is implemented and pump-probe studies are undertaken in order to study polarization-dependent gain dynamics in bulk semiconductor optical amplifiers (SOAs) and their application to gain and polarization switching. The polarization dependence of the gain compression shows that the co-polarized case, in which the pump and probe are TE-polarized, is the optimum for gain switching. When injecting light polarized at $45^{\circ}$, an additional contribution due to the presence of rotating fields is observed, and the cross-polarized case is found to be the most promising for polarization switching. The potential for all-optical switching based on nonlinear polarization rotation (NLPR) induced in the SOA is then assessed through characterization of the dynamics of the state of polarization. It is demonstrated that by careful selection of the orientation of a linear polarizer in front of the detector, it is possible to reduce the recovery time from 100 to $5 \mathrm{ps}$. The improvement comes about from the selection of the intraband fast gain recovery dynamics. Polarization switching offers potentially faster switching times compared to gain switching as well as an extinction ratio improvement of $\sim 10 \mathrm{~dB}$.
\end{abstract}

Index Terms-All-optical switching, cross-gain modulation, nonlinear polarization rotation (NLPR), pump-probe, semiconductor optical amplifiers.

\section{INTRODUCTION}

A $\mathrm{T}$ present, the speed and capacity of optical communications are limited by the optical/electronic conversion devices used for switching functions. The limit for these electronic elements due to the so-called electronic bottleneck is $40 \mathrm{Gbit} / \mathrm{s}$ [1]. Currently, $40 \mathrm{Gbit} / \mathrm{s}$ systems have just reached the stage where they are commercially available, while the next generation of $160 \mathrm{Gbit} / \mathrm{s}$ systems is currently under active study [2]. At these data rates, all-optical processing becomes a necessity.

Despite the fact that the nonlinearities of semiconductor optical amplifiers (SOAs) limit their use as amplifiers, these phenomena are fundamental to realizing many attractive all-optical

Manuscript received September 12, 2007; revised January 31, 2008. Current version published October 24, 2008. This work was supported by Enterprise Ireland.

S. Philippe and A. L. Bradley are with the School of Physics, Trinity College Dublin, Dublin 2, Ireland (e-mail: philipps@tcd.ie).

B. Kennedy was with the School of Electronic Engineering, Dublin City University, Glasnevin, Dublin 9, Ireland. He is now with the Optical and Biomedical Engineering Laboratory, University of Western Australia, Australia.

F. Surre is with the School of Physics, Trinity College Dublin, Dublin 2, Ireland, and the School of Electronic Engineering, Dublin City University, Glasnevin, Dublin 9, Ireland.

P. Landais is with the School of Electronic Engineering, Dublin City University, Glasnevin, Dublin 9, Ireland.

Digital Object Identifier 10.1109/JLT.2008.922326 signal-processing applications. Bit-rate capabilities in excess of $100 \mathrm{Gbit} / \mathrm{s}$ have been reported using a variety of schemes [3]. All these techniques are limited by their sensitivity to the polarization of the optical input signals; therefore polarization effects in SOAs must be thoroughly characterized in order to implement and optimize the various switching schemes.

The polarization sensitivity of SOAs, due to different gain and refractive indexes in the TE and TM modes, is well known [4]. Considerable efforts have been made to reduce this sensitivity, using strain and waveguide engineered structures. Gain anisotropy of less than $2 \mathrm{~dB}$ has been achieved, though this is only guaranteed under injection of a single continuous-wave (CW) signal. The change of the state of polarization and the gain, experienced by a CW or pulse injected signal as it travels through the SOA, are both altered when a second signal, pulsed or not, is injected into the device. This phenomenon, nonlinear polarization rotation, can potentially be used for high-speed alloptical switching applications [5]-[8]. While a large amount of work focusing on its applications has been performed, the underlying physical mechanisms are not thoroughly understood. In order to optimize nonlinear polarization switching and determine its limitations, the mechanisms governing the dynamics of the change in polarization and its recovery must be determined.

In this paper, polarization-dependent gain dynamics as well as the dynamics of the change of the state of polarization (SOP) are studied in the picosecond regime. The results are then used to determine the best working points for gain switching and polarization switching.

This paper is organized as follows. In Section II, the device under test and experimental setup are presented, while in Section III, the measurement techniques are described. The experimental results are given in Section IV for injection of signals polarized along the eigenmodes of the device and Section V for injected signals linearly polarized at $45^{\circ}$. Lastly, in Section VI, conclusions are drawn.

\section{EXPERIMENTAL SET-UP}

Cross-gain and cross-phase modulation [5]-[7], [9] are the most common all-optical wavelength conversion schemes based on SOAs. They usually include some fiberized elements, which makes it very difficult to control and characterize the state of polarization of both the injected and the transmitted optical signals. In the context of a characterization of polarization effects, a free-space setup, as shown in Fig. 1, is ideal so that all SOPs can be controlled and preserved for observation.

As the orientation of each linearly polarized input signal is varied, and includes the co-polarized pump and probe combi- 


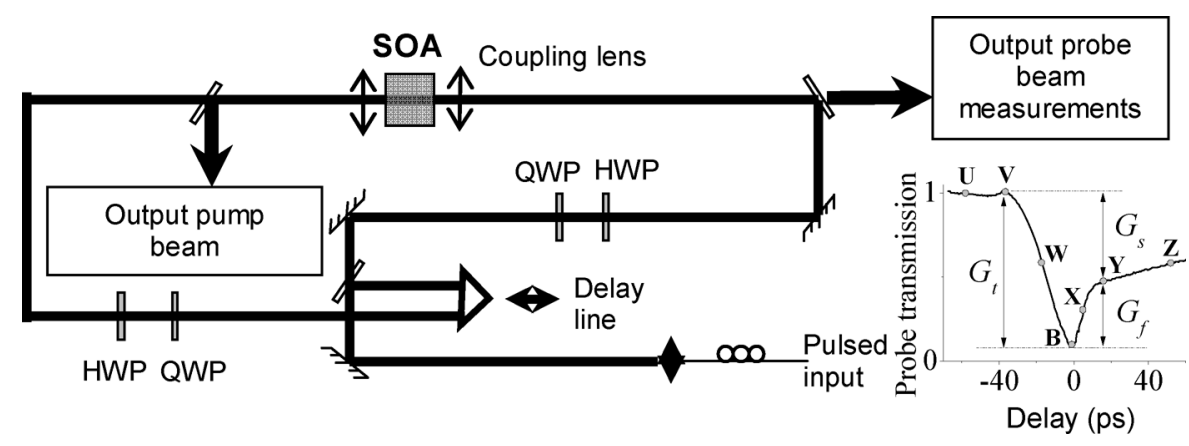

Fig. 1. Experimental setup. The inset shows typical decay, gain parameters, and values of delay used for polarization measurements.

nation, then the probe cannot be separated using a polarizer, as has been the case in previously reported co-propagation setups. Therefore, a contra-propagation is used, in which the two input signals travel in opposite directions and thus can easily be separated using a beamsplitter. Since the main focus of this paper is to assess the potential of gain compression and nonlinear polarization rotation (NLPR) for optical telecommunications at data rates over $100 \mathrm{~Gb} / \mathrm{s}$, the dynamics are studied in the picosecond regime. The input is provided by a fiber-based femtosecond laser with a repetition rate of $82 \mathrm{MHz}$. After passing through a $1 \mathrm{~nm}$ spectral width filter, the pulse width of the input signal is $2.5 \mathrm{ps}$ and its wavelength set at $1580 \mathrm{~nm}$. The probe and pump pulses are coherent. One of them is delayed with respect to the other using a variable delay line with a time increment of $530 \mathrm{fs}$. The signal acquisition and the displacement of the delay line are automated and the time integrated probe intensity is recorded as a function of the pump-probe time delay.

The device under test was a commercial $1.5 \mathrm{~mm}$ long bulk InGaAsP/InP traveling wave SOA. In this setup, it is biased at $350 \mathrm{~mA}$ and temperature controlled at $20^{\circ} \mathrm{C}$. Under these experimental conditions, it exhibits a gain peak of $20 \mathrm{~dB}$ at $1580 \mathrm{~nm}$, higher in TM than in TE by less than $2 \mathrm{~dB}$. Further details of the device under test can be found in [4]. The noise generated by the amplified spontaneous emission (ASE) is reduced using a $1580 \mathrm{~nm}$ filter, and the signal is then detected using an infrared photodiode and a lock-in technique.

\section{MEASUREMENTS}

There are two methods for investigating polarization effects in an SOA using pump-probe experiments, both of which are presented in this paper. Monitoring the probe transmitted intensity as a function of delay for different pump and probe input states of polarization allows us to study the polarization dependence of the gain dynamics, while rotating a polarizer in front of the photodiode allows us to characterize the change in the probe state of polarization at different pump-probe delays. These studies are complementary as the device response to different input states of polarization gives information on the underlying physics of the gain dynamics, whereas on the other hand, the polarization-dependent detection differentiates between pure gain compression and polarization effects.

A typical probe intensity decay is shown in the inset of Fig. 1. Two components in the probe transmission recovery can be seen. The delay between the pump and probe pulses is set to zero when the influence of the pump is the greatest. Before $\mathrm{V}$, the probe leaves the SOA before the pump enters it. Between $\mathrm{V}$ and $\mathrm{B}$, the pulses overlap at different positions inside the SOA. After the zero delay, the pump leaves the SOA before the probe enters and recovery of the device gain is observed. A fast component, due to intraband effects, occurs between zero delay and Y, followed by a slower component due to interband recombination [10]. With picosecond pulse widths, we expect significant contributions of both interband and intraband effects [11].

Different gain parameters are measured, giving information on the relative importance of intraband and interband effects as a function of the input states of polarization and injected pump energy. Three different gain parameters are extracted from the transmission curves, as shown in the inset of Fig. 1. The total gain compression $G_{t}$ is measured between $\mathrm{V}$ and $\mathrm{B}$. The slow gain recovery component $G_{s}$, measured after Y, corresponds to the part of the signal recovering by slower interband effects. The fast gain recovery component $G_{f}$, measured by subtracting the slow gain recovery from the total gain compression, corresponds to the probe gain recovery by faster intraband effects.

Although the pulse width of $2.5 \mathrm{ps}$ used here does not allow for the time scales of spectral hole burning and carrier heating $(\mathrm{CH})$ (typically $<500 \mathrm{fs}$ ) to be resolved, the polarization dependence of the intra- and interband dynamics can be determined by examining the relative contributions of the fast and slow components of the probe transmission as a function of the pump-probe time delay. With a similar device using a co-propagation pump-probe scheme, a time scale of 2 ps has been reported for $\mathrm{CH}$ [10]. However, the convolved lifetimes can be extracted, indicating their combined effects on the probe signal. We define the fall time as the $10 \%$ to $90 \%$ fall time between V and $\mathrm{B}$ and the fast recovery time as the $10 \%$ to $90 \%$ rise time between $\mathrm{B}$ and $\mathrm{Y}$. The slow time scale is found by an exponential fit of the slow recovery recorded after Y.

In order to specifically study the change of the output probe SOP, a polarizer is placed in front of the detector and the signal detected as a function of the angle of the polarizer, providing a direct characterization of the probe SOP. The signal is detected using a lock-in detection technique. By repeating these measurements at different pump-probe time delays-for example, as shown in Fig. 2, the dynamics of the nonlinear polarization rotation can be assessed and the contribution of intraband and interband effects investigated. No measurements can be taken at 


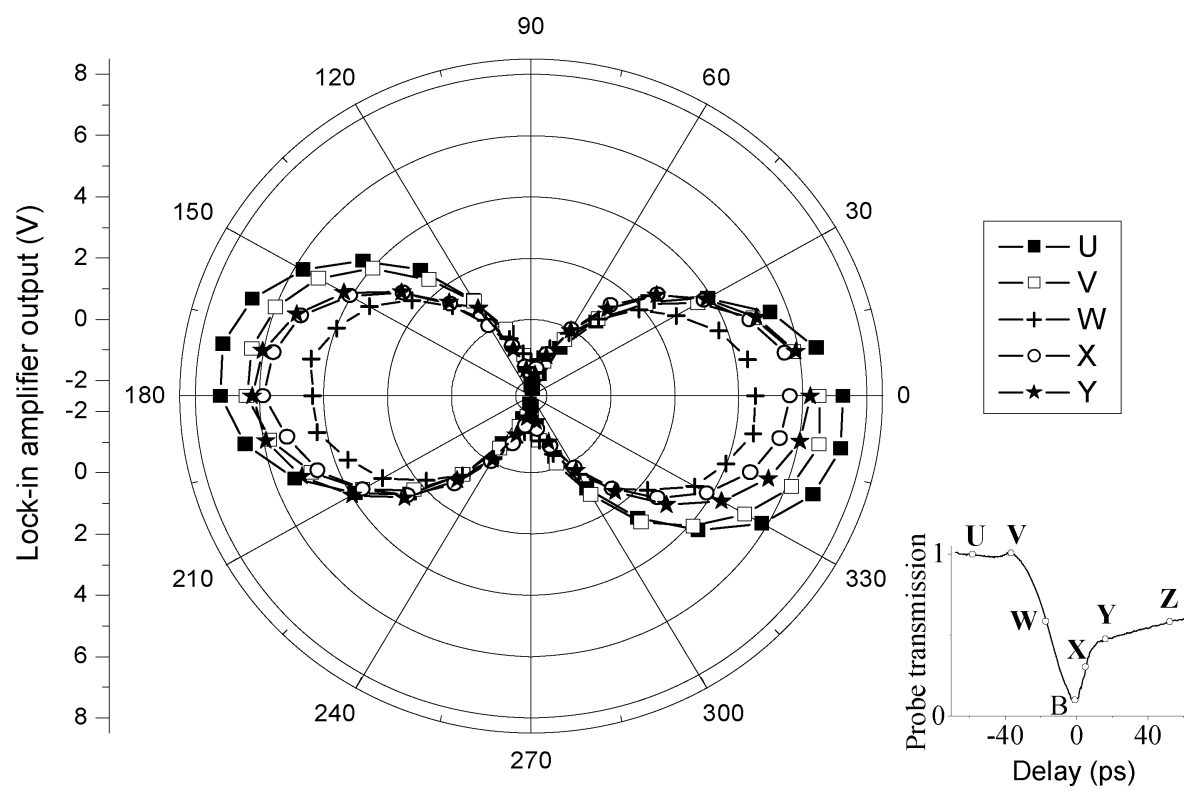

Fig. 2. Output intensity as a function of polarizer angle, probe TE, $12 \mathrm{fJ}$ pulse energy and pump TE, $875 \mathrm{fJ}$ pulse energy. Inset shows delays at which polar plots were recorded.

B because of oscillations due to a coherent artifact and the low level of the transmitted signal. The values measured at $\mathrm{Y}$ and $\mathrm{Z}$ are found to be very close and therefore, for clarity only, data recorded at delay Y are shown in the polar plots.

\section{InJeCtion Along the EIgENMOdes of THE Device}

\section{A. Polarization Rotation}

The first set of experiments investigates the polarization-dependent gain dynamics for pump and probe signals injected along the eigenmodes of the SOA. A key issue is to determine whether or not there is any modification of the SOP of a signal injected along an eigenmode. An example is shown in Fig. 2 for a TE polarized probe with $12 \mathrm{fJ}$ pulse energy and a TE polarized pump with $875 \mathrm{fJ}$ pulse energy. The pump pulse energy can be varied from 12 to 875 fJ. The pump and probe wavelength are set at $1580 \mathrm{~nm}$ at the peak of the SOA gain spectrum. Polarization resolved measurements at six different delays are recorded and the SOP of the probe remains almost unchanged, linear and close to $0^{\circ}$ orientation, for all pump pulse energies and pump-probe delays. At the highest pump power, a small rotation of the probe state of polarization can be seen. It is always less than $10^{\circ}$ and too low to be used for polarization switching. This small modification of the probe polarization may be due to the emergence of eigenmodes other than the fundamental under these conditions of injected signals in the SOA [6]. The negative values on the polar plot correspond to when the polarizer is at $90^{\circ}$ with respect to the transmitted probe signal and a small amount of amplified spontaneous emission modulated by the chopped probe signal is detected. The change in transmitted probe intensity at different pump-probe delays corresponds to the probe gain compression and is greater at high pump energies, as expected.

\section{B. Polarization Dependent Dynamics}

The pump and probe wavelength are again set at $1580 \mathrm{~nm}$ at the peak of the SOA gain spectrum. The probe transmission is recorded as a function of pump-probe delay for a range of pump pulse energies (6-975 fJ), while the probe pulse energy is kept constant at $12 \mathrm{fJ}$, below the gain saturation energy. The data are taken for the different combinations of probe and pump SOPs, probe and pump TE polarized (TETE), probe and pump TM polarized (TMTM), probe TE pump TM (TE $\left.\mathrm{Tr}_{\mathrm{pr}} \mathrm{TM}_{\mathrm{pu}}\right)$, and probe TM pump TE $\left(\mathrm{TM}_{\mathrm{pr}} \mathrm{TE}_{\mathrm{pu}}\right)$. The legend of each figure gives the probe SOP, followed by that of the pump. When the pump and probe polarizations are set along the eigenmodes of the device, they travel through it with their polarization unchanged. Therefore, in all cases, an output polarizer is aligned with the input probe polarization in order to isolate the probe signal from the ASE.

The results for two values of the pump pulse energy (24 and 732 fJ) can be seen in Fig. 3(a) and (b). A clear polarization dependence of the probe transmission is observed. In all cases, larger gain compression is observed for co-polarized pump and probe signals. In Fig. 3(a), the onset of the decrease in the probe transmission occurs at earlier time delays for the co-polarized cases TETE (TMTM) than for the cross-polarized cases $\mathrm{TE}_{\mathrm{pr}} \mathrm{TM}_{\mathrm{pu}}\left(\mathrm{TM}_{\mathrm{pr}} T E_{\mathrm{pu}}\right)$. This is due to the fact that the effect of the pump on the probe is greater when pump and probe pulses are polarized along the same eigenmode, and therefore the pump has to traverse less of the SOA to induce gain compression of the probe signal. An additional feature is observed around $-30 \mathrm{ps}$ when the pump and probe pulses overlap close to the output facet of the SOA. It can also be noted that this feature is strongly polarization dependent. For high pump powers, there is an increase in the probe transmission in the TMTM case, whereas the feature is absent in the $\mathrm{TE}_{\mathrm{pr}} \mathrm{TM}_{\mathrm{pu}}$ case at all pump energies. Modeling is under way to study the origin of this effect, which most likely arises from modification 


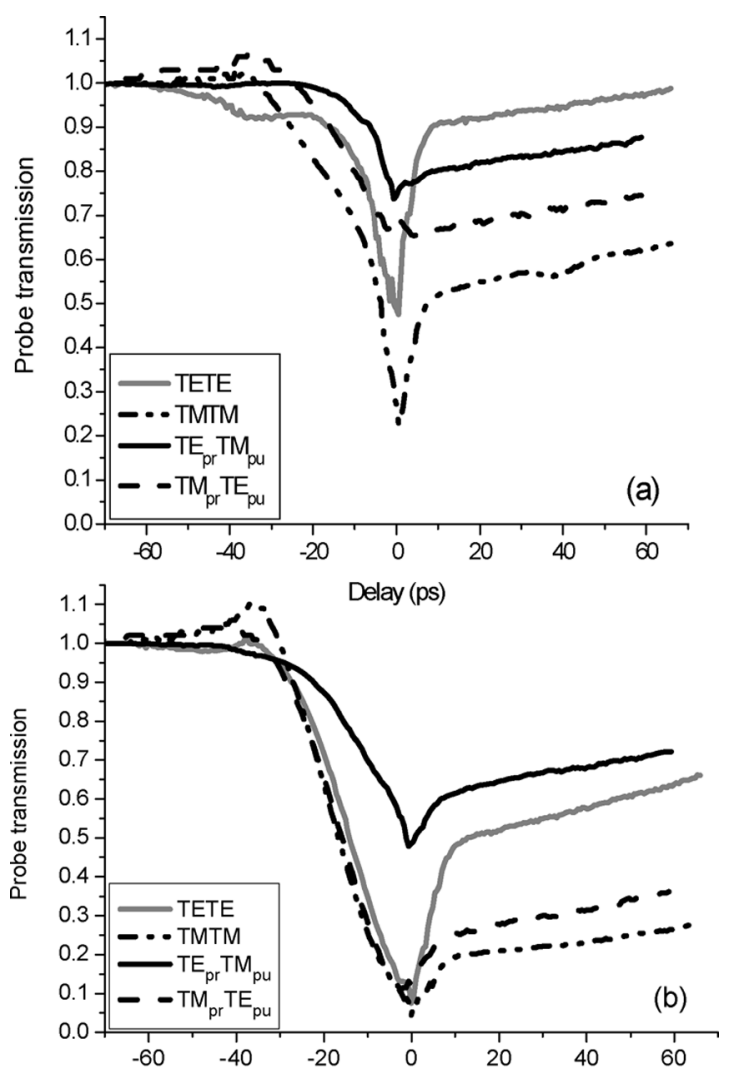

Fig. 3. Transmitted pump signals as a functuon of delay for the different co- and cross-polarized cases. $\mathrm{E}_{\text {probe }}=12 \mathrm{fJ}$, (a) $\mathrm{E}_{\text {pump }}=24 \mathrm{fJ}$, and (b) $\mathrm{E}_{\text {pump }}=$ $732 \mathrm{fJ}$.

of the carrier distribution along the device [12]. The depletion of carriers at a given position inside the device can lead to a decrease in the amount of ASE traveling from that position and thus increase the carrier density at other positions along the SOA.

In all cases, the probe gain compression increases as the pump energy is increased. For each probe-pump polarization combination, the total gain compression (measured between $\mathrm{V}$ and $\mathrm{B}$ ) as a function of pump energy is shown in Fig. 4. As the pump energy increases, the gain compression increases, as would be expected from carrier depletion effects. At higher pump energies, the gain compression stabilizes in the co-polarized cases. Clearly higher gain compression is achieved by pumping and probing the same eigenmode. When pumping orthogonal to the probed mode, much higher pump energy is needed to reach the same gain compression. For the probe TM polarized, a gain compression of $\sim 7 \mathrm{~dB}$ can be achieved by either injecting a $24 \mathrm{fJ}$ TM polarized pump or a $732 \mathrm{fJ}$ TE polarized pump, giving a cross-gain compression ratio of $3.3 \%$. Similarly, for the probe TE polarized, a gain compression of $\sim 2.5 \mathrm{~dB}$ is obtained with a $12 \mathrm{fJ}$ TE polarized pump or a $732 \mathrm{fJ}$ TM polarized pump, giving a cross-gain compression ratio of $1.6 \%$. Therefore, cross-gain compression from one mode to the other is low but increases with pump energy; and cross-gain compression from the TE pump to TM probe is higher than from the TM pump to TE probe.

The separate contributions of the fast and slow components to the probe gain recovery are presented in Figs. 5 and 6, respectively. Clearly, from Fig. 5, the fast gain recovery compo-

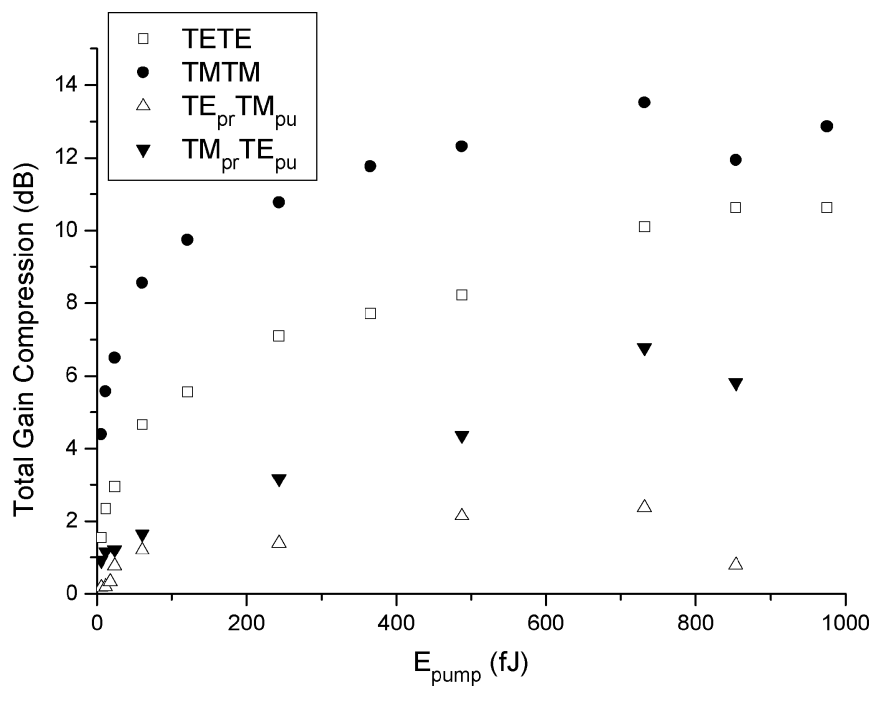

Fig. 4. Total gain compression as a function of pump pulse energy for the different input polarization cases.

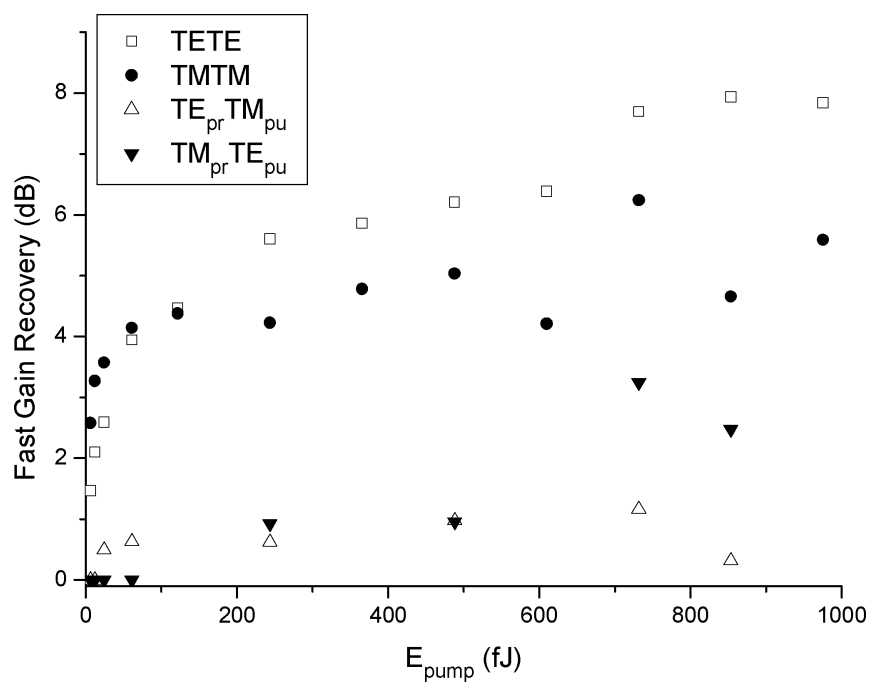

Fig. 5. Fast gain recovery as a function of pump pulse energy for the different input polarization cases.

nent is most evident when pump and probe are co-polarized. The fast recovery dominates the probe transmission recovery for the TETE case, where only a small level of slow gain recovery remains, as can be seen in Fig. 6. A fast component is also evident for the TMTM case, but there is a greater contribution of the slow gain recovery in that case.

For a $2.5 \mathrm{ps}$ pulse, the fast gain recovery component is mainly attributed to carrier cooling [11], [13], [14]. In the TETE case, it remains significant even at high pump pulse energies, whereas in the TMTM case, its contribution to the recovery is less significant as the pump pulse energy increases. Fast gain recovery values are close for TETE and TMTM cases. TETE seems slightly larger by about $1 \mathrm{~dB}$, but this is within the resolution of the experiment at these low transmission values. However, the total gain compression is higher in the TMTM case, meaning that a smaller proportion of carrier density has recovered by carrier cooling than in the TETE case. These experimental results have been successfully modeled by 


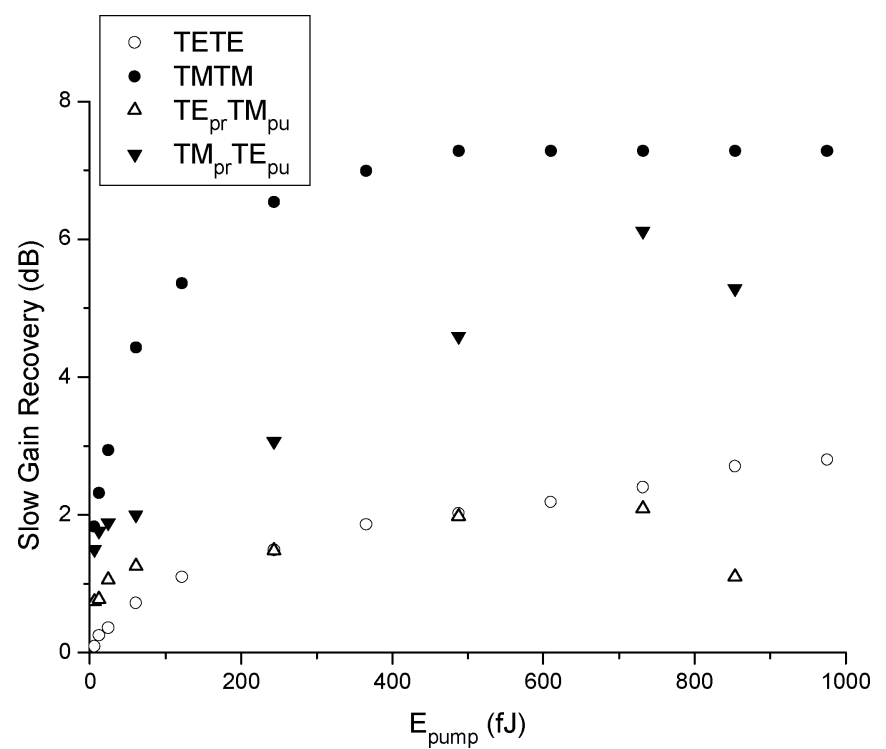

Fig. 6. Slow gain recovery as a function of pump pulse energy for the different input polarization cases.

numerical simulation based on the carrier density rate equation and taking into account the polarization dependence of the intraband gain compression [15].

Considering now the slow gain recovery (Fig. 6), different behavior is observed for each pump-probe polarization combination. The discrepancy between the TM mode and TE mode gain behavior is most likely due to strain effects [16], [17]. This is commonly present in bulk devices, even for what is usually considered a negligible lattice mismatch [16], and has the consequence of removing the degeneracy of the light and heavy hole valence bands. The transitions associated with TE and TM modes are different, with TM transitions involving light holes while TE transitions involve a mixture of light (25\%) and heavy (75\%) holes [5], [18]. As a consequence, in all cases an increase in slow gain recovery with pump energy is observed, as the conduction band is depleted by the pump, whatever its polarization may be. However, the amount of slow gain recovery differs due to the different transitions involved for each eigenmode from the conduction band to the heavy hole and light hole valence bands. In the case of a TM probe, the slow gain recovery is always higher when pumping in TM than in TE. The difference increases rapidly with pump energy as the light holes are quickly depleted by the TM pump. The difference then decreases after saturation of the light hole valence band is reached for the pump TM polarized but not for the TE polarized pump where only a minority of the transitions involve the light hole valence band.

In the co-polarized cases, the pump signal depletes the carriers by the same transitions used for probe amplification. The slow gain recovery is much higher in the TM mode than in TE mode. As can be seen in Fig. 6, the pump energy needed to achieve the same amount of slow gain recovery is lower for TM than for TE; this is consistent with a lower density of states of the light hole valence band. The TM slow gain recovery reaches a constant value of $7 \mathrm{~dB}$ above a $400 \mathrm{fJ}$ pump energy while the TE slow gain recovery keeps increasing slowly with pump energy but only reaches $2.8 \mathrm{~dB}$. One possible explanation is that the saturation is mainly experienced by the light hole valence

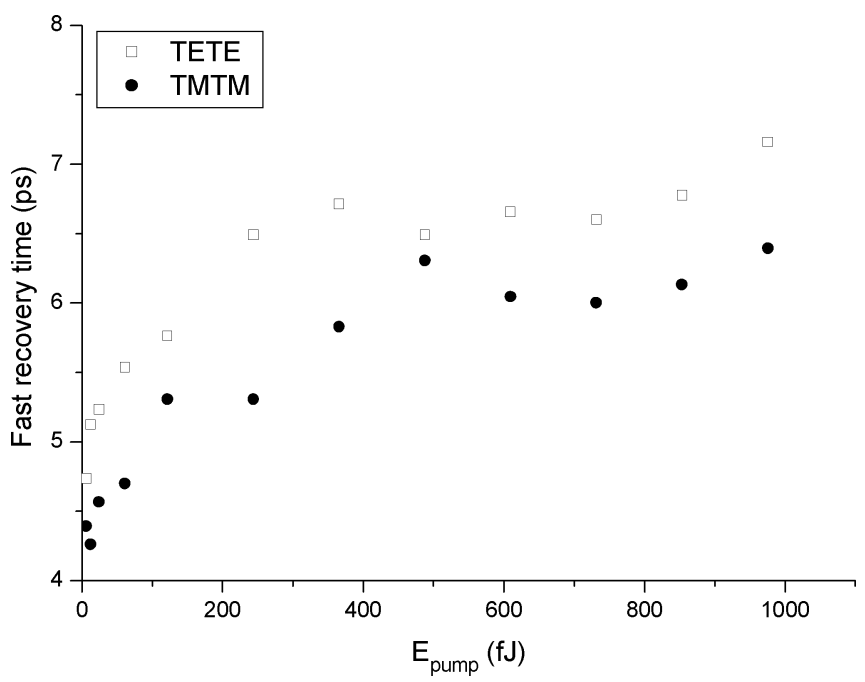

Fig. 7. Fast recovery time as a function of pump pulse energy for the different input polarization cases.

band, so in the TMTM case, a high gain compression occurs, whereas in the TETE case, the saturation of the light hole valence band affects at most $25 \%$ of the transitions.

As mentioned above, in the cross-polarized cases, a greater cross-gain saturation from the TE pump to TM probe than from the TM pump to TE probe is observed. The TE transitions involve mainly heavy holes but also $25 \%$ light holes, which can lead to a significant reduction of light holes at higher pump powers and thus a decrease in the TM probe gain. On the other hand, the decrease in light holes due to a TM pump will only concern at most $25 \%$ of the holes associated with the TE probe transitions.

At low pump energies, the slow gain recovery is higher for $\mathrm{TE}_{\mathrm{pr}} \mathrm{TM}_{\mathrm{pu}}$ than TETE, as the light holes are depleted by the TM pump while the TE pump energy is not high enough to deplete significantly the light hole valence bands. As the pump power is increased, the TM pump saturates the light hole valence band, whereas the slow gain recovery then keeps slowly increasing when the pump is TE polarized.

The characteristic times associated with the fast and slow recovery components were also examined. The fall time corresponds to the pump and probe pulses overlapping inside the SOA and quickly stabilizes around $22 \mathrm{ps.}$

Fig. 7 shows the fast recovery time for the co-polarized cases. The fast recovery time increases with pump energy and is slightly lower in TMTM than in TETE, with a minimum of $4.4 \mathrm{ps}$ at $6 \mathrm{fJ}$ in TMTM and a maximum of $7.2 \mathrm{ps}$ at $975 \mathrm{fJ}$ in TETE. The fast component corresponds to a convolution of the fast and slower effects. As the slow timescale measured in the TETE case is slightly higher than in the TMTM, a slightly slower fast recovery time in TETE can be expected even for equal time constants of the intraband effects. Comparing Figs. 7 and 4, it can be seen that the range of energies over which the fast recovery time increases corresponds to an increase in gain compression, while the slow time scale was found to be constant. Therefore, the increase in the fast time scale with increasing pump energy can be attributed to the increase in gain compression [10]. 


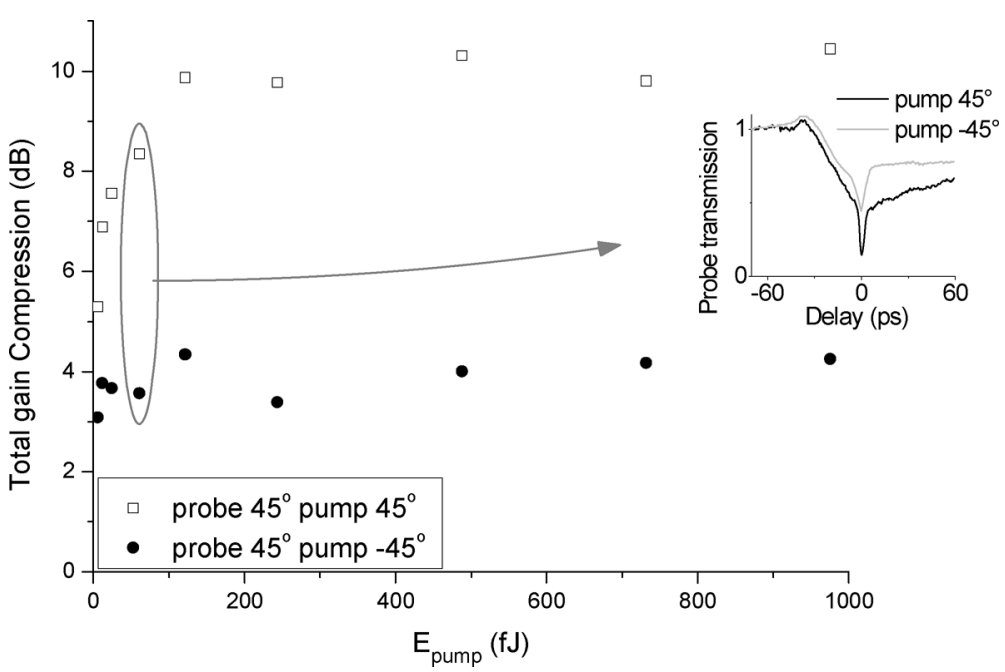

Fig. 8. Total gain compression as a function of pump pulse energy, probe linearly polarized at $45^{\circ}$, pump linearly polarized at $45^{\circ}$ and $-45^{\circ}$. Decays for $E_{\text {pump }}=$ $61 \mathrm{fJ}$ shown in the inset.

The characteristic time for the slow gain recovery component decreases rapidly with increasing pump pulse energy up to $50 \mathrm{fJ}$. After this, it stabilizes with values between 100 to 300 ps depending on pump and probe polarizations. A decrease of the recovery time with injected optical power has been observed by other groups [12], [19] and attributed to the effect of the ASE on gain dynamics.

\section{Gain Switching}

For gain switching applications, the polarization dependence of the cross-gain modulation has to be taken into account. The pump and probe beams are generally set along the eigenmodes of the device and travel through it with their polarization unchanged. However, as was discussed above, the performance of the switch would be greatly affected by the state of polarization of the input signals. It is obvious from Fig. 3 that the co-polarized configuration is most interesting for cross-gain modulation, with a faster component present even at lower pump energies and overall higher gain compression. Although the TMTM case displays a higher total gain compression and would therefore give a higher extinction ratio, it is dominated by the slower interband effects. Therefore the TE mode would give the best performances for ultrafast gain switching.

\section{INJECTION AT $45^{\circ}$}

\section{A. Gain Dynamics}

The results and discussion of Section IV dealt with the case where the pump and probe signals are injected along the TE or TM eigenmode of the device. As the signals' SOPs remained unchanged, the observed dynamics were unaffected by any NLPR contribution. Therefore, for polarization switching, the probe input state of polarization must be set at a different orientation so that it will be altered as it travels through the device. In this case, the combined effects of the polarization-dependent gain and the change in the probe SOP have to be taken into account. In this section, the gain dynamics are studied for signals linearly polarized at orientations of $45^{\circ}$ and $-45^{\circ}$ with respect to the eigenmodes of the device so that the same amount of power is injected in each eigenmode. Since a contra-propagation configuration is used, the cross-polarized case is probe $45^{\circ}$, pump $45^{\circ}$, while probe $45^{\circ}$ and pump $-45^{\circ}$ is co-polarized. The co/cross-polarization only refers to the states of polarization of the injected signals, as this will change for both the pump and the probe as they propagate through the device. In this case, there is no polarizer in front of the detector so that the total gain compression is measured independently of any NLPR effect. The impact of the pump pulse on the probe transmission is found to be different in the co- and cross-polarized cases, with a stronger gain compression in the cross-polarized case, as can be seen in Fig. 8.

The total gain compression, shown in Fig. 8, increases rapidly with pump energy in the cross-polarized case and stabilizes around $10 \mathrm{~dB}$, while it stays almost constant at $4 \mathrm{~dB}$ in the co-polarized case. The contributions of both intraband and interband effects to the gain recovery are always larger in the cross-polarized case. The slow gain recovery increases with pump energy, due to carrier depletion, while the additional fast gain recovery decreases slightly. In both cases, pumping at $45^{\circ}$ and $-45^{\circ}$, intraband and interband effects contribute similarly to the total gain recovery without one dominating it. This remains true over the whole range of injected pump energy, unlike what is observed in the femtosecond regime where intraband effects dominate at low pump energy [20].

In the cross-polarized case (probe $45^{\circ}$, pump $45^{\circ}$ ), the total gain compression and fast gain recovery values are similar to the co-polarized TETE and TMTM cases. The slow gain recovery values are close to that obtained in the TMTM case. The co-polarized (probe $45^{\circ}$, pump $-45^{\circ}$ ) total gain compression values are closer to the $\mathrm{TE}_{\mathrm{pr}} \mathrm{TM}_{\mathrm{pu}}$ and $\mathrm{TM}_{\mathrm{pr}} \mathrm{TE}_{\mathrm{pu}}$ cases, while the slow gain recovery is closer to the TE polarized probe data (TETE and $\mathrm{TE}_{\mathrm{pr}} \mathrm{TM}_{\mathrm{pu}}$ ). There is a fast component present, even at low pump energies, as was the case for TETE and TMTM, but with a lower amplitude.

The fall time and slow recovery time measured are similar to those obtained when injecting light polarized along the eigenmodes of the device. The fast recovery time stays constant as the pump energy is increased. It is shorter in the cross-polarized (probe and pump $45^{\circ}$ ) case than in the co-polarized case, at about 
2.5 and $4.5 \mathrm{ps}$, respectively. The $4.5 \mathrm{ps}$ fast recovery time is similar to what is observed at low pump energy when injecting along the eigenmodes of the device. However, the $2.5 \mathrm{ps}$ time scale is faster than any time scale measured when pumping and probing along the eigenmodes of the device. As this time corresponds to the pulse width, the dynamic may be even faster but cannot be resolved. The shorter fast recovery time and higher gain compression make the cross-polarized case the most promising for polarization-switching applications.

Taking a simplified approach, in which the $45^{\circ}$ pump and probe signals are resolved into equal contributions in the TE and TM modes, it might have been expected that the gain parameters and recovery dynamics would be similar for the crossand co-polarized $45^{\circ}$ signals. The fact that the behavior is different in both cases is a consequence of the change in the SOPs of the pump and probe signals as they propagate in the SOA waveguide. They can evolve from their initial linear polarization to elliptical and circular as they propagate through the device. This introduces additional considerations-for example, if counter-circularly polarized pump and probe signals are used, angular momentum considerations lead to a decoupling of the spin populations and the signals do not couple to the same conduction band to light/heavy hole valence band transitions [21], [22]. Therefore, different behavior could be expected for cocircular and countercircular pump-probe configurations. In our case, the SOPs of both the pump and probe signals would be changing continuously at they travel through the SOA. Thus at different positions inside the device, we have different combinations of co-polarized, cross-polarized, circular, and elliptical polarizations. Therefore, when injecting at $45^{\circ}$, the level of gain compression is not a straightforward combination of the TE and TM gain dynamic behavior.

\section{B. Polarization Rotation}

The evolution of the output probe SOP is now measured for the $45^{\circ}$ cross-polarized case, which was found to be the most promising in the previous section. The probe pulse energy is $12 \mathrm{fJ}$, and two different values of pump energy, 12 and $875 \mathrm{fJ}$ are investigated. First, we monitor the change in output probe SOP as a function of pump-probe delay. The lock-in amplifier output voltage, proportional to the probe transmitted intensity, as a function of the output polarizer orientation is shown in Fig. 9. Propagation through the SOA causes the polarization to become elliptical (U). For a low power pump pulse [Fig. 9(a)] the onset of gain compression (W) causes the probe polarization to become less elliptical, approximating a linear polarization. It remains in this SOP as the probe gain recovers with an almost constant orientation. In the high pump pulse energy case [Fig. 9(b)], again at delay position U, the output probe has an elliptical SOP. As the gain compression increases, it becomes almost circular at a delay of $-8 \mathrm{ps}(\mathrm{W})$ and becomes linear as the device starts to recover (X) and maintains this polarization beyond $55 \mathrm{ps}(\mathrm{Y}, \mathrm{Z})$. The fact that the polarization stays quasi-linear as the device recovers indicates that the recovery of the probe SOP is dominated by the slower interband effects. These results are consistent with measurements performed with injected continuous wave laser beams, in which interband effects dominate and the injection of a pump beam causes the output probe SOP to become linear at this bias current.
A full recovery of the output probe intensity due to the combined effects of birefringence and gain effects was demonstrated in the femtosecond regime [20]. We investigate how similar results may be obtained in the picosecond regime even though the contribution of interband effects is much higher.

Examination of Fig. 9 clearly shows that very different polarization-switching performances can be achieved depending on the orientation of the output polarizer. As shown in Fig. 10(a), placing a polarizer at $0^{\circ}$ deteriorates the performance by increasing the minimum probe transmission from 0.2 to 0.5 , while placing a polarizer at $45^{\circ}$ improves the performance by decreasing the minimum transmission from 0.2 to 0.01 and by dramatically speeding up the intensity recovery. In other words, the extinction ratio is degraded by $4 \mathrm{~dB}$ in the first case but improved by $13 \mathrm{~dB}$ in the second case in comparison with the case where there is no output polarizer. Furthermore, the intensity recovers to its initial value in approximately $5 \mathrm{ps}$ in the second case. It is important to remember here that recovery of the intensity does not imply full recovery of the gain. The trends observed at higher pump power depicted in Fig. 10(b) are the same. Yet again the introduction of a polarizer at $45^{\circ}$ increases the switching extinction ratio achievable and results in a faster recovery, though clearly the benefits are less than with a low pump. These measurements clearly show that even though the recovery of the polarization is dominated by the slower interband effects, a fast recovery and full recovery of the probe transmitted intensity can still be achieved by careful selection of the output polarizer orientation.

For comparison, the decays corresponding to the most promising gain-switching and polarization-switching configurations are shown in Fig. 11. The optimum gain switching is achieved for co-polarized TE pump and probe, with a pump pulse energy of $875 \mathrm{fJ}$. The $20 \mathrm{~dB}$ extinction ratio measured for polarization switching is higher than that of $10.5 \mathrm{~dB}$ for gain switching, and the $12 \mathrm{fJ}$ pump energy needed is smaller than the $875 \mathrm{fJ}$ in the gain-switching case. For polarization switching, the $10 \%$ to $90 \%$ rise time between 0 ps and $\mathrm{Y}$ is approximately $5 \mathrm{ps}$, slightly faster than the $6.7 \mathrm{ps}$ measured for gain switching. The most interesting effect is that we can isolate the fast component of the probe signal recovery using a polarizer at the detector by virtue of the fact that the probe SOP has changed, even though full recovery of both the gain and SOP is determined by the slower interband effects. Using polarization switching, the full recovery of the probe intensity can be obtained within $5 \mathrm{ps}$, while the minimum full recovery time measured for gain switching is about 100 ps.

\section{CONCLUSIONS}

Both the polarization resolved dynamics and the dynamics of NLPR in a bulk SOA were studied using a free-space contrapropagation configuration. A series of pump-probe experiments were performed, with co- or cross-polarized pulses, injected along the eigenmodes of the device and linearly polarized at $45^{\circ}$. The gain compression and characteristic time scales were found to be dependent on the pump and probe SOP and pump pulse energy. When injecting along the eigenmodes of the device, the cross-polarized data are dominated by interband effects and show a low cross-saturation between the modes. It was found that the prevalence of the fast intraband effects in the 

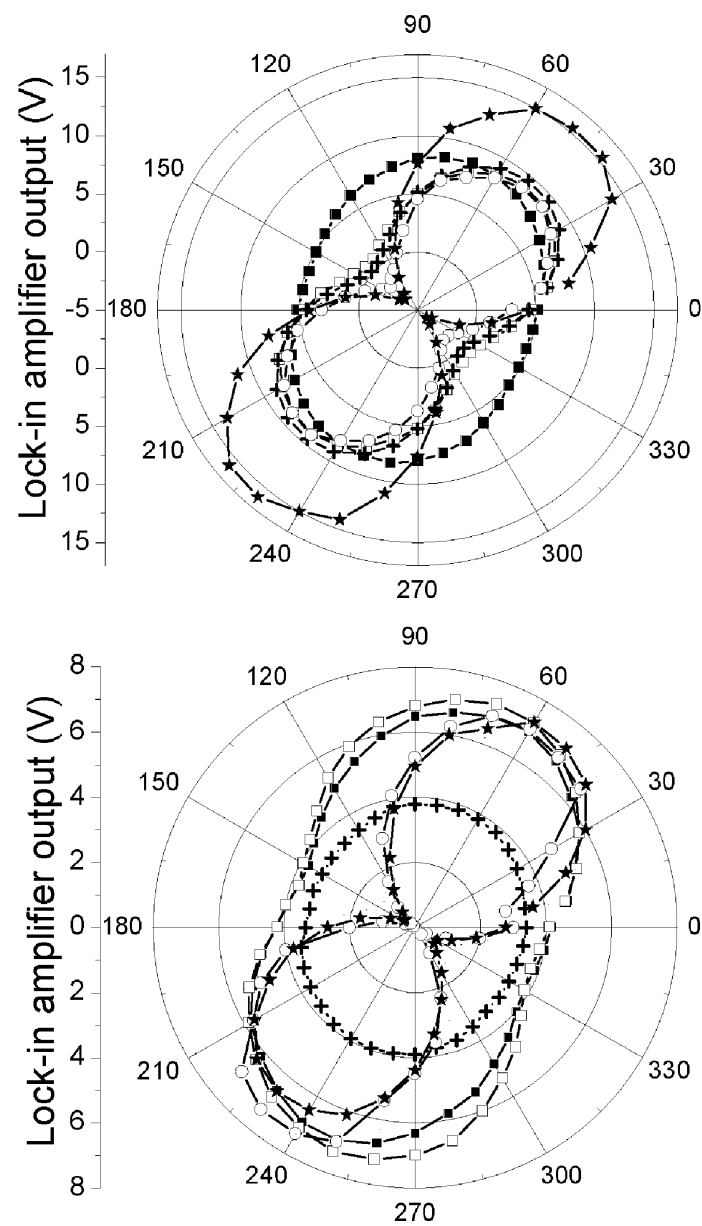

(a)
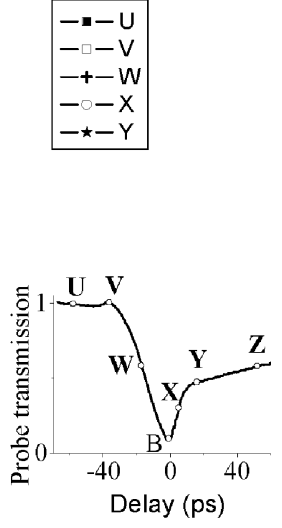

(b)

Fig. 9. Output intensity as a function of polarizer angle. (a) Probe $12 \mathrm{fJ}$ and pump $12 \mathrm{fJ}$. (b) Probe $12 \mathrm{fJ}$ and pump $875 \mathrm{fJ}$.
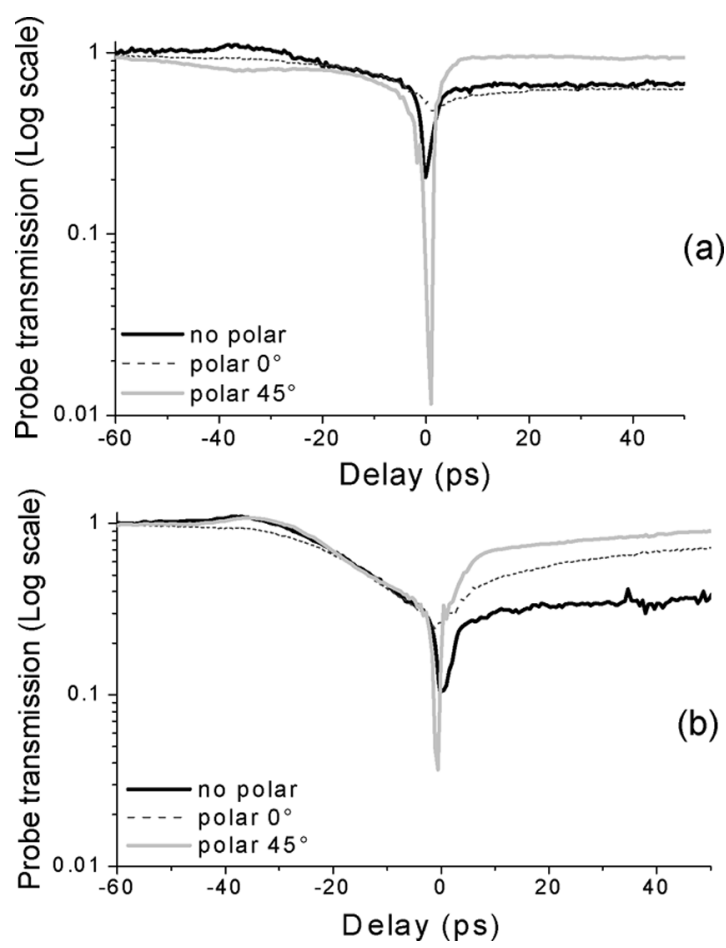

Fig. 10. Polarization resolved decays, pump and probe linearly polarized at 45 . (a) Probe $12 \mathrm{fJ}$ and pump 12 fJ. (b) Probe 12 fJ and pump 875 fJ.

TETE case shows the highest potential for ultrafast all-optical gain switching. In order to assess the potential for polarization

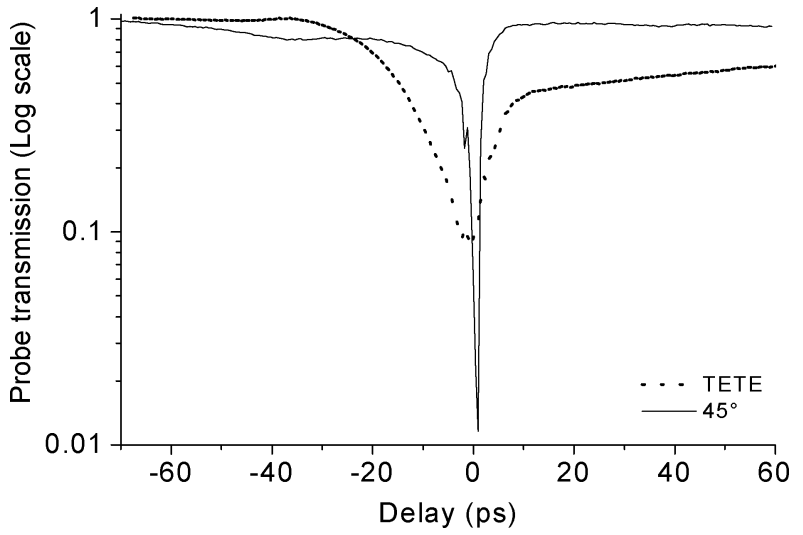

Fig. 11. Comparison of gain switching (TE pump and probe signals with $\left.\mathrm{P}_{\text {probe }}=12 \mathrm{fJ}, \mathrm{P}_{\text {pump }}=875 \mathrm{fJ}\right)$ and gain and polarization switching $\left(45^{\circ}\right.$ injection, output polarizer at $45^{\circ} \mathrm{P}_{\text {probe }}=12 \mathrm{fJ}, \mathrm{P}_{\text {pump }}=12 \mathrm{fJ}$ ).

switching, the gain recovery when injecting linearly polarized signals with a $45^{\circ}$ orientation was studied. The cross-polarized case, with both pump and probe signals linearly polarized at $45^{\circ}$ and an output polarizer oriented at $45^{\circ}$, is the most promising configuration for polarization switching, with a full recovery of the probe intensity within $5 \mathrm{ps}$. Full recovery of the probe SOP is governed by interband dynamics. However, the slow recovery of the output probe intensity due to the slowly recovering gain can be compensated for by the increased transmitted intensity through an output polarizer due to the change in polarization. 
Polarization switching offered $9.5 \mathrm{~dB}$ improvement in extinction ratio for lower pump pulse energy, in comparison to the optimum gain-switching configuration. In addition, the complete recovery of the probe intensity was shortened from 100 to $5 \mathrm{ps}$. Therefore, we demonstrated that it is possible to select the gain recovery mechanism and potentially achieve faster switching speeds.

\section{REFERENCES}

[1] R. T. R. Almeida, K. Potratz, L. C. Bueno, M. E. V. Segatto, E. Oliveira, and L. C. Calmon, "Addressing the electronic bottleneck to virtual topology design of optical networks," in Proc. 2003 Microw. Optoelectron. Conf. (IMOC 2003)/SBMO/IEEE MTT-S Int., vol. 2, pp. 925-930.

[2] H. G. Weber, R. Ludwig, U. Feiste, C. Schmidt, C. Schubert, J. Berger, E. Hilliger, M. Kroh, and T. Yamamoto, "High-speed all-optical signal processing in optical communication systems," in Proc. Tech. Dig. Pacific Rim Conf. Lasers Electro-Opt. (CLEO), 2002, p. 610.

[3] K. E. Stubkjaer, "Semiconductor optical amplifier-based all-optical gates for high-speed optical processing," IEEE J. Sel. Topics Quantum Electron., vol. 6, pp. 1428-1435, 2000.

[4] B. F. Kennedy, S. Philippe, P. Landais, A. L. Bradley, and H. Soto, "Experimental investigation of polarisation rotation in semiconductor optical amplifiers," Proc. Inst. Elect. Eng. Optoelectron., vol. 151, pp. 114-118, 2004.

[5] D. Lenstra, Y. Liu, M. T. Hill, G.-D. Khoe, and H. J. S. Dorren, "Nonlinear polarization rotation in semiconductor optical amplifiers: Theory and application to all-optical flip-flop memories," IEEE J. Quantum Electron., vol. 39, pp. 141-148, 2003.

[6] H. Soto, J. C. Dominguez, D. Erasme, and G. Guekos, "Demonstration of an all-optical switch using cross-polarization modulation in semiconductor optical amplifiers," Microw. Opt. Technol. Lett., vol. 29, no. 3, pp. 205-209, 2001.

[7] M. F. C. Stephens, M. Asghari, R. V. Penty, and I. H. White, "Demonstration of ultrafast all-optical wavelength conversion utilizing birefringence in semiconductor optical amplifiers," IEEE Photon. Technol. Lett., vol. 9, pp. 449-451, 1997.

[8] B. F. Kennedy, S. Philippe, F. Surre, A. L. Bradley, and P. Landais, "Investigation of optimum wavelength converter based on nonlinear polarisation rotation in a bulk SOA," IET Optoelectron., vol. 1, pp. 55-60, 2007.

[9] Y. Liu, E. Tangdiongga, Z. Li, S. Zhang, H. De Waardt, G. D. Khoe, and H. J. S. Dorren, " $80 \mathrm{Gbit} / \mathrm{s}$ wavelength conversion using semiconductor optical amplifier and optical bandpass filter," Electron. Lett., vol. 41, pp. 487-489, 2005.

[10] L. Occhi, Y. Ito, H. Kawaguchi, L. Schares, J. Eckner, and G. Guekos, "Intraband gain dynamics in bulk semiconductor optical amplifiers: Measurements and simulations," IEEE J. Quantum Electron., vol. 38, pp. 54-60, 2002.

[11] P. Borri, S. Scaffetti, J. Mork, W. Langbein, J. M. Hvam, A. Mecozzi, and F. Martelli, "Measurement and calculation of the critical pulsewidth for gain saturation in semiconductor optical amplifiers," Opt. Commun., vol. 164, pp. 51-55, 1999.

[12] R. Gutierrez-Castrejon, L. Schares, L. Occhi, and G. Guekos, "Modeling and measurement of longitudinal gain dynamics in saturated semiconductor optical amplifiers of different length," IEEE J. Quantum Electron., vol. 36, pp. 1476-1484, 2000.

[13] Y. Takahashi, A. Neogi, and H. Kawaguchi, "Polarization-dependent nonlinear gain in semiconductor lasers," IEEE J. Quantum Electron., vol. 34, pp. 1660-1672, 1998.

[14] B. N. Gomatam and A. P. DeFonzo, "Theory of hot carrier effects on nonlinear gain in GaAs-GaAlAs lasers and amplifiers," IEEE $J$. Quantum Electron., vol. 26, pp. 1689-1704, 1990.

[15] S. Philippe, A. L. Bradley, F. Surre, P. Landais, B. Kennedy, and M. Martinez-Rosas, "Polarization dependence of non-linear gain compression factor in semiconductor optical amplifier," Opt. Express, vol. 16, pp. 8641-8648, 2008.

[16] W. Wang, K. Allaart, and D. Lenstra, "Semiconductor optical amplifier gain anisotropy: Confinement factor against material gain," Electron. Lett., vol. 40, pp. 1602-1603, 2004.

[17] T. D. Visser, H. Blok, B. Demeulenaere, and D. Lenstra, "Confinement factors and gain in optical amplifiers," IEEE J. Quantum Electron., vol. 33, pp. 1763-1766, 1997.

[18] E. P. O'Reilly and A. R. Adams, "Band-structure engineering in strained semiconductor lasers," IEEE J. Quantum Electron., vol. 30, pp. 366-379, 1994.
[19] F. Girardin, G. Guekos, and A. Houbavlis, "Gain recovery of bulk semiconductor optical amplifiers," IEEE Photon. Technol. Lett., vol. 10, pp. 784-786, 1998

[20] H. Ju, S. Zhang, D. Lenstra, H. De Waardt, E. Tangdiongga, G. D. Khoe, and H. J. S. Dorren, "SOA-based all-optical switch with subpicosecond full recovery," Opt. Express, vol. 13, pp. 942-947, 2005.

[21] A. Leitenstorfer and A. Laubereau, "Ultrafast electron-phonon interactions in semiconductors: Quantum kinetic memory effects," in Semiconductors and Semimetals: Ultrafast Physical Processes in Semiconductors, K. T. Tsen, Ed. New York: Academic, 2001, vol. 67.

[22] W. H. Louisell, Quantum Statistical Properties of Radiation. New York: Wiley, 1973.

Severine Philippe received the B.Sc. degree (first-class honors) in applied sciences from Dublin Institute of Technology, Dublin, Ireland, in 2002 and the Ph.D. in physics from Trinity College Dublin in 2007.

She is currently a Postdoctoral Researcher with the Semiconductor Photonics Group, School of Physics, Trinity College. Her research interests include ultrafast all-optical switching, semiconductor optical amplifiers, and rare earthdoped fibers.

Dr. Philippe received a scholarship from IRCSET.

A.L. Bradley received the B.Sc. degree (first-class honors) from University College Dublin, Dublin, Ireland, in 1992 and the M.Sc. and Ph.D. degrees from Trinity College, Dublin, in 1994 and 1998, respectively.

Her doctoral work involved the study of cavity polaritons using nonlinear optical technique as part of the European project SMILES. She went on to work on the development of $\mathrm{GaP}$ microcavity light-emitting diodes and later $\mathrm{GaN}$ devices under the European project AGETHA. She has been a Lecturer with the School of Physics, Trinity College, since 2001. Her current research interests include semiconductor devices for optical communications and novel structures of controlling semiconductor light emission.

B. Kennedy received the B.Sc. (honors) and Ph.D. degrees in electronic engineering from Dublin City University, Dublin, Ireland, in 2001 and 2006, respectively.

From March 2006 to December 2007, he was a full-time Lecturer in the Electrical Engineering Department, Universidad de Santiago de Chile. He is currently with the Optical and Biomedical Engineering Laboratory, University of Western Australia.

F. Surre (S'00-A'03-M'03) received the B.E degree in electronic engineering and the M.E degree in microwave and optical communications from Ecole $\mathrm{Na}-$ tionale Supérieure d'Electrotechnique, d'Electronique, d'Informatique, d'Hydraulique, Toulouse, France, in 1998. He received the Ph.D. degree in electronic engineering from the Institut Polytechnique de Toulouse in 2003.

His doctoral work involved study of the modeling and design of multiscale circuits in electromagnetism. Between 1998 and 1999, he was a Research Engineer with the Physics Laboratory, École Nationale de la Statistique et de l'Administration Économique, France, where he was responsible for opto/microwave measurements. After one year working on quasi-optical systems at Queen Mary College, University of London, U.K., he joined the School of Physics, Trinity College, Dublin, Ireland, and the Research Institute for Networks and Communications Engineering, Dublin City University, Dublin. His research interests include polarization dependence of ultrafast dynamics in semiconductor optical amplifiers (SOAs), noise in SOAs, and generation and guiding of terahertz waves.

Pascal Landais (M'02) received the Ph.D . degree in applied physics from the Ecole Nationale Supérieure des Télécommunications, Paris, France, in 1995.

His early work concerned the study of bistable and self-pulsating semiconductor lasers as all-optical functional components in fiber telecommunication systems. In 1996, he joined the Physics Department, Trinity College, Dublin, Ireland, where he developed low-coherence semiconductor lasers for data storage and stabilized Fabry-Perot lasers. Between 1997 and 1999, he was involved in research activities on microcavity light-emitting diodes under the European project SMILED. Between 1999 and 2000, he was a Development Manager with CeramOptec Ltd, Ireland. Since 2001, he has been a Lecturer with the School of Electronics Engineering, Dublin City University, Dublin. His current research topics include components for optical communication systems and terahertz generation.

Dr. Landais is a TMR Marie Curie Fellow and one of the Irish representatives to COST 288. 